\title{
Microheaters fabricated from a conducting composite
}

\author{
Liyu Liu, Suili Peng, Xize Niu, and Weijia Wen ${ }^{\text {a) }}$ \\ Department of Physics, The Hong Kong University of Science and Technology, Clear Water Bay, Kowloon, \\ Hong Kong
}

(Received 27 September 2006; accepted 21 October 2006; published online 1 December 2006)

\begin{abstract}
The authors report the fabrication of microheaters based on a conducting composite of silver microparticles embedded in polydimethylsioxane (PDMS). Experimental results show that the microheaters exhibit good performance in temperature rise and decay characteristics, with localized heating at targeted spatial domains. A unique feature of the microheater here is its excellent integration capability with biocompatible PDMS and other polymer materials, with potential microchip applications for bioprocessing and chemical reactions. (c) 2006 American Institute of Physics. [DOI: 10.1063/1.2400065]
\end{abstract}

Microfluidic chips have demonstrated their significant application potentials in microbiological processing and chemical reactions, with the goal of developing monolithic and compact chip-sized multifunctional systems. Heat generation and thermal control are critical in some of the biochemical processes. Hence microheaters are widely employed in different microchips, such as heating for nanoparticle productions in microfluidic chemical reactors, ${ }^{1}$ providing thermal convection in biological processes, ${ }^{2}$ vaporizing liquid in microchannels, ${ }^{3}$ etc. In the past decade, various microheaters have been developed based on different working principles. It has been reported that microheaters could be manufactured by laser direct-writing technology on alumina substrate, ${ }^{4}$ sputtering thin films of platinum ${ }^{5}$ and nickel $^{6}$ on silicone or glass plate, as well as the micro-Peltier junctions, ${ }^{7}$ etc.

The miniaturization of microheaters can avoid heating of the entire chip during processing, ${ }^{8,9}$ which would seriously limit independent thermal operations, e.g., microscale polymerase chain reaction (PCR) needs three sections with different temperatures within a single chip. ${ }^{10}$ The challenge nowadays is to develop thermal devices with miniature dimensions and localized heating characteristics, which are highly integrable with base materials. In this letter, we report the fabrication of microheaters based on silver and polydimethylsioxane (PDMS) composite and demonstrate the ease of fabrication, integration, and localized temperature control.

PDMS is widely used in microfluidic systems, due to its biocompatibility and ease of processing., ${ }^{8,11,12}$ The material used to fabricate our microheaters is made by synthesizing PDMS with microsized silver particles $(1-2 \mu \mathrm{m}$ in diameter). The two materials are mixed and grinded together at the silver concentration of $86.3 \%(w / w)$ and subsequently filled into photoresist molds (fabricated via soft lithography) with square helical grooves. After baking at $70{ }^{\circ} \mathrm{C}$ for $2 \mathrm{~h}$, the composite solidified and the molds were rinsed away by acetone, leaving only solid patterns on the substrate. Pure PDMS were overlaid on the patterns and lifted off the substrate after it has hardened.

Figure 1 shows a cartoon and a scanning electron microscopy (SEM) picture (inset) of the microheater. The helical-patterned microheater is sealed and supported by a

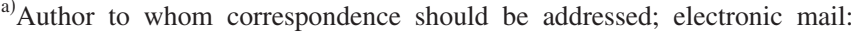
phwen@ust.hk
}

PDMS base and protrudes from the surface upward. Since the composite material is conductive, when the two outstretched wires are connected to positive and negative voltages, electrical current and hence heat will be generated. In the experiments, various molds with widths ranging from 25 to $100 \mu \mathrm{m}$ were used to make the conducting stripes. The height of all the heater stripes is $14.4 \mu \mathrm{m}$. From the inset to Fig. 1, one can see that the width of the heater stripe is $\sim 25 \mu \mathrm{m}$ (for taking the SEM image, the microheater is not sealed with a PDMS layer) and the dimension of the microheater is about $200 \times 200 \mu \mathrm{m}^{2}$. Since the composite and base materials (PDMS) are rubberlike with good flexibility, our testing results show the microheater to be operational even when the whole chip was mildly bent.

In order to examine the heating capability of the microheaters, an infrared (IR) camera (FLIR Systems trademark, model Prism DS) was employed to detect both the heat images and the local temperatures. The IR camera was placed right over the microheater to record the thermal characteristics when the microheater was subjected to different applied voltages. By using this infrared sensing technique, we were able to obtain accurate temperature readings as well as comprehensive thermal distribution patterns. In our experiment, the relationship between temperature and applied voltage was determined by focusing the IR camera on the central helical range of the microheater. The measured results for a heater with $\sim 75 \mu \mathrm{m}$ widths of stripes are shown in Fig. 2,

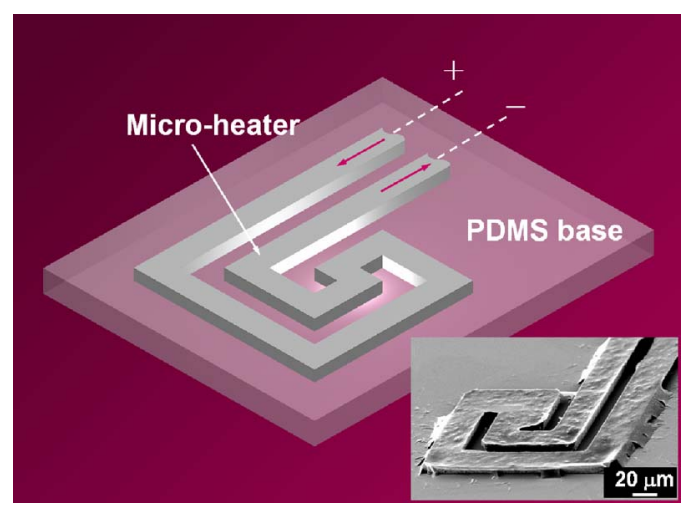

FIG. 1. (Color online) Schematic illustration of the microheater. The threedimensional helical-patterned structure is made from a silver-microparticlesPDMS - composite. Inset: SEM picture of the microheater whose linewidth is $25 \mu \mathrm{m}$. 


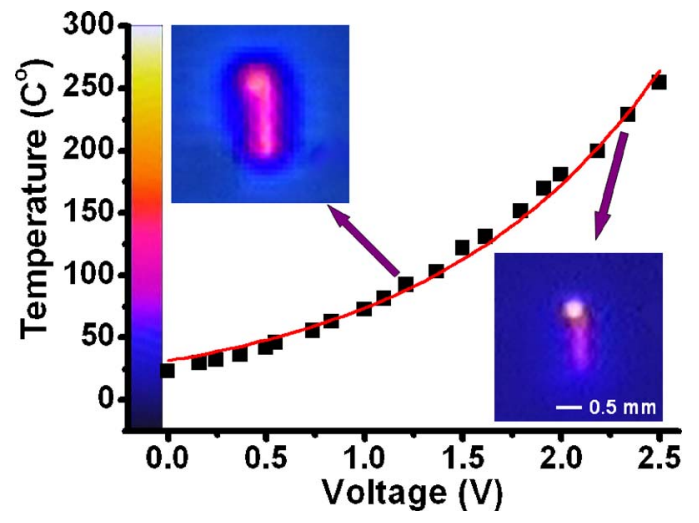

FIG. 2. (Color online) Temperature of the microheater's central heating part plotted as a function of the input voltage. The two insets are IR pictures showing the thermal distributions at specific applied voltages. The bright spot on the right panel is a high temperature region with a temperature of $\sim 250{ }^{\circ} \mathrm{C}$.

from which we can see that the temperature rises monotonically from room temperature with increasing applied voltage. The relationship can be well fitted by an exponential. The maximum temperature is seen to reach about $250{ }^{\circ} \mathrm{C}$ when the applied voltage is $2.5 \mathrm{~V}$. Two actual IR images of the microheater taken at different voltages are shown in the insets. On the left panel, the heating distribution has a rectangular shape with a broad thermal distribution, while on the right panel one can observe localized heating distribution (the bright spot that is $\sim 400 \times 400 \mu \mathrm{m}^{2}$ in area) where the temperature of the microheater was raised up to $250{ }^{\circ} \mathrm{C}$. These thermal distribution pictures show the heated area to be much larger than the size of the microheater, with lower temperatures extending much further beyond the heater than the higher temperatures, as necessitated by heat conduction. The relatively small area of the high temperature region means that the microheater can be useful for sample annealing or reaction carried out locally, such as those of biochip and microchemical reactors.

The temperature rise and decay characteristics of the microheater are important features in the microheater's response to applied voltage. We have measured the response characteristics of the microheaters by applying a square voltage pulse. In the experiment, a single square pulse (the power on duration is $100 \mathrm{~s}$ as shown by the dash line in Fig. 3 ) is applied to the microheater while the thermal variations are recorded with the IR video camera. The temperature variation versus time and applied voltages are derived from individual image frames by photoimage processing. The relationship thus obtained is plotted in Fig. 3. It clearly shows that the temperature of the microheater increased very rapidly when the pulse was applied, with a rising time on the order of a few seconds, and then the temperature increase became much slower until it reached a stabilized plateau. When the pulse was turned off, the temperature initially decreased quickly, followed by a slower variation until it resumed the room temperature. The temperature rise and decay times are listed in a table shown in Fig. 3. Here the rise and decay response times of the microheater are defined at the points where the temperature reaches $80 \%$ of the stabilized temperature. It is noted from the table in Fig. 3 that the rise time becomes longer with increasing applied voltage up to $2.0 \mathrm{~V}$ but decreases after that. On the other hand, the decay time decreases monotonously with increasing voltage, implyDownloaded 28 Jun 2007 to 143.89.104.110. Redistribution subject

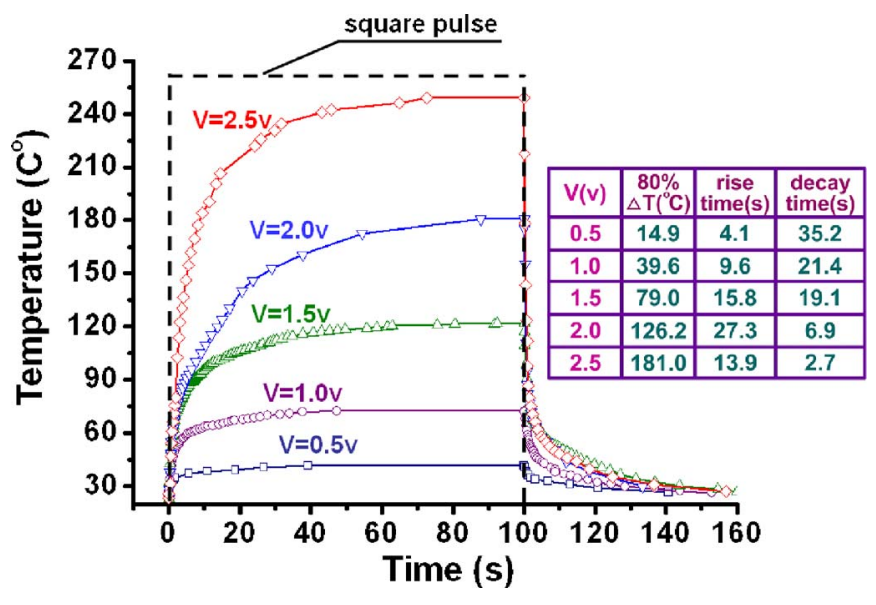

FIG. 3. (Color online) Time dependent temperature variations of the microheater under a $100 \mathrm{~s}$ square voltage pulse with indicated applied voltages. The right inset tabulates the temperature rise and decay times, defined at points where the temperature is $80 \%$ of the stabilized value.

ing that once the pulse is suspended, the heat tends to dissipate faster from higher temperatures. From these results, it follows that if two pulses are combined and applied in sequence, i.e., a long low voltage pulse following immediately a short high voltage pulse, a certain stable temperature can be attained quickly.

Since the present microheater is PDMS based, it can be easily integrated with other PDMS or polymer components, thereby exhibiting more flexibility as compared to the conventional microheaters. Figure 4(a) shows an example on how to design and assemble a thermal digital display panel (or display cell) combined with seven isolated microheaters implanted into a PDMS base material. As indicated in Fig. 4(a) each microheater is connected to two metallic electrodes, sharing a common ground with the others and independently controlled. Figure 4(b) shows the IR images of the digitally controlled display cell. With proper combinations of signals, heat infrared trails are seen to compose the digits from 1 to 9 . With programed digital operations, the display is also able to freely change from one digit to any other within seconds.

In summary, microheaters based on silver-powderPDMS composite have been fabricated and successfully demonstrated. With soft-lithographical technology, easy fabrication with low cost is possible for mass production. In addition, it is integrable with other PDMS microdevices, which would enable the microheaters to be applied in areas

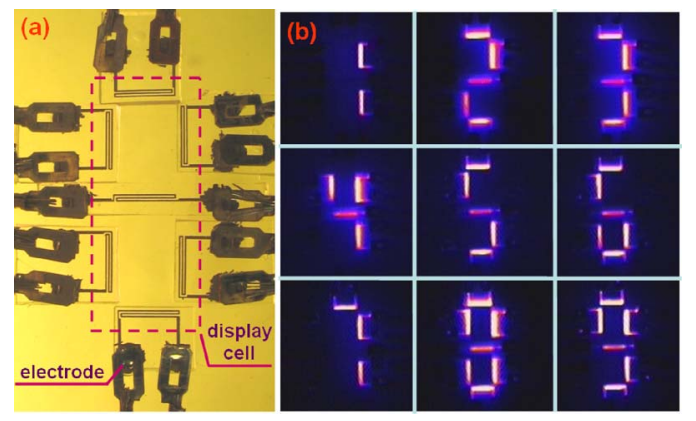

FIG. 4. (Color online) (a) Integration of multiple microheaters to form a thermal display. In (b) the infrared images of the digits are displayed, demonstrating the ease and flexibility of operations.

to AIP license or copyright, see http://apl.aip.org/apl/copyright.jsp 
such as microbioprocessing, microfluidic analysis, microchemical reaction, etc.

The authors acknowledge the financial support for this work form Hong Kong NSFC/RGC joint research Grant No. HKUST 605/04.

${ }^{1}$ A. J. deMello, Nature (London) 442, 394 (2006).

${ }^{2}$ M. Henning and D. Braun, Appl. Phys. Lett. 87, 183901 (2005).

${ }^{3}$ Z. Yin and A. Prosperetti, J. Micromech. Microeng. 15, 1683 (2005).

${ }^{4}$ T. Tong, J. Li, Q. Chen, J. Longtin, S. Tankiewicz, and S. Sampath, Sens. Actuators, A 114, 102 (2004).
${ }^{5}$ G. Chung, Sens. Actuators, A 112, 55 (2004).

${ }^{6}$ H. F. Arate, H. Noji, and H. Fujita, Appl. Phys. Lett. 88, 083902 (2006).

${ }^{7}$ G. Maltezos, M. Johnston, and A. Scherer, Appl. Phys. Lett. 87, 154105 (2006).

${ }^{8}$ E. M. Lucchetta, M. S. Munson, and R. F. Ismagilov, Astron. Tsirk. 6, 185 (2006).

${ }^{9}$ S. C. Tadic, G. Dernick, D. Juncker, G. Buurman, H. Kropshofer, B. Michel, C. Fattinger, and E. Delamarche, Lab Chip 4, 563 (2004).

${ }^{10}$ A. J. deMello, Nature (London) 422, 28 (2003).

${ }^{11}$ L. Liu, X. Niu, W. Wen and Ping Sheng, Appl. Phys. Lett. 88, 173505 (2006).

${ }^{12}$ X. Niu, L. Liu, W. Wen, and P. Sheng, Phys. Rev. Lett. 97, 044501 (2006). 